\title{
Physics Essay: Particle Nature of Light and the Speed of Light
}

\author{
Zifeng $\mathrm{Li}^{1}$ \\ ${ }^{1}$ Petroleum Engineering Department, Yanshan University, Hebei, China \\ Correspondence: Zifeng Li, Petroleum Engineering Department, Yanshan University, China. E-mail: zfli@ ysu.edu.cn
}

Received: October 7, 2015 Accepted: October 21, 2015 Online Published: October 24, 2015

doi:10.5539/apr.v7n6p49 URL: http://dx.doi.org/10.5539/apr.v7n6p49

\begin{abstract}
What is the nature of light? Particle or wave? How fast is the speed of light? These are all basic questions of physics. From points of philosophy and observation, it is demonstrated that the nature of light is a kind of particle, and many photons' group behavior is of characteristics similar to the wave. Reflection, diffusion, refraction and transmission of light are all phenomenon of matter's attraction, absorption and re-emission of photons. Until now, there is no accurate means of measuring the speed of light. For the speed of starlight relative to the earth, the closer to the earth, the closer to the speed of light emitted by a light source on the earth relative to the earth. Physics field is composed of space and tiny particles.
\end{abstract}

Keywords: light, particle, the speed of light, measure, field

\section{Introduction}

The nature of light has always been the focus of the physical sector concerned. However, it has been controverted but it has been in a situation rarely being studied (10000 Science crux physics editorial committee, 2009). The study on the nature of light enables people to explore the microscopic world and the evolution of the universe, and reveal the unknown phenomenon better. For the description of the nature of light, in the theoretical circles there are three theories: (1) wave, (2) particle, (3) wave-particle duality. Associated with the nature of light, the speed of light (Kragh, 2006; Li et al., 2007; Li, 2008; Smeulders, 2008), and the essence of the field have always caught people's attention. This paper, from points of philosophy and observation, give the demonstration that the nature of light is a kind of particle, and then explore the speed of light measurement, finally outlined the nature of the field.

\section{Three Traditional Theories on the Nature of Light}

\subsection{Light is a Kind of Wave}

In the spread process, mechanical wave has phenomenon of interference, diffraction and so on. So does light. According to the phenomenon analogy, it can infer that the nature of light is a kind of wave, rather than a kind of particle.

\subsection{Light is a Kind of Particle}

In the light irradiation, the phenomenon of an object emitting electrons is called the photovoltaic effect. Therefore, it can infer that light is a kind of particle, rather than the wave.

\subsection{Wave-Particle Duality}

Such doctrine believes that the light is a wave, but also a particle. The wave talked about here is a kind of probability wave, only meaningful to a large number of photons. The particle talked about here refers to its non-continuity.

(1)The effect of the single photon often manifests particle nature; the effect of a large number of photons often manifests wave nature (Fan \& Feng, 2007). (2) Light of high frequency often show the particle nature; and light of low frequency often show the wave nature. (3) Light often behaves the wave nature when propagation; and often behaves the particle nature when interacts with the material.

\section{The Essence of Light is Particle Whose Group Behavior with the Characteristics similar to Wave}

The world consists of space and matter distributed in space. Matter distributes in the form of particles in space. Particles can be divided into different levels, for example, the sun, the earth, car, atom, electron, photon and so on. The nature of light is a kind of particle-photon. It is a level of the physical structure. Photon is material with mass and energy. Photon is composed of much smaller particles. Because people still observe the world by light, there is no way clearly observing the material even smaller than the photon; however starlight passed the sun nearby is attracted 
by the gravity of the sun, which proved that substances even smaller than the photon exist. When people invented means of observing the world by particles much smaller than the photon, the structure of the photon can be observed.

As the nature of light is particle, then how to explain the wave characteristics of light? Luminous objects are regularly emitting a series of photons. For a single photon, there is only the particle nature, and the absence of the wave characteristics (the internal structure of the photon must also have the wave characteristics, not be discussed here). For the light, that is, in terms of a series of photons, is of both particle nature and characteristics similar to the wave (Panarella, 1987). For example, it was figuratively, for the parade unit in the Tian An Men Square, a single person or a car can be seen as a particle that composes a phalanx being of several rows, being of cycles; a phalanx can be seen as a particle group, one by one phalanx has regular movement, and has characteristics similar to the wave.

In order to distinguish wave motion nature of the mechanical wave, the characteristics of groups of photons similar to the characteristics of mechanical wave is called the characteristics similar to the wave.

\section{Reflection, Scattering, Refraction and Transmission of Light}

Reflection, scattering, refraction and transmission of light are processes that photons are attracted, absorbed and re-emitted by the particles within the object when encounter an object. This process takes time. The degree and way of different objects to attract, absorb and re-emit of light are different. Attracted by objects, light can deviate from the original direction of movement. After object absorbs light, the light can be re-emitted in the same frequency, can also be-emitted in other frequency, can also be released in other forms of mater, and can also exist inside the object. Re-emit contains reflection to the object emitting incident light, refraction and scattering inside the object, and transmission through an object. For example: black body absorbs light more and emits light less, in the irradiation of light, whose temperature raises and that releases heat rays; the solar cells absorb the light energy to change into electrical energy and store electrical energy up; transmission of glass is good, while the iron is poor.

\section{Measurement of the Speed of Light}

The speed of photon relative to light source is the escape velocity of the photon. inside the light source revolves around a core at the high speed. When its tangential velocity is greater than or equal to the escape velocity, the photon will sent out from the light source. As the speed of satellites to reach or exceed the escape velocity of the earth, the satellite will send out from the earth.

In 1607, Galileo conducted the earliest experiment of measurement of the speed of light. Galileo's approach is to allow two persons were standing in two mountains separated by a mile away, each person holding a lamp, the first person raised the lamp first, the second person raised his own lamp as soon as he saw the first person's lamp, the time interval from the first person raised lamp to he had seen the light of the second person is time that light propagates two miles away. However, light travels so fast that this method will not work. But Galileo's experiment lifted the curtain of study on the speed of light in human history.

Until modern times, there has been a variety of methods to measure the speed of light, but these methods are based on the reflection method of measuring the return speed of light. There is no approach measuring one-way speed of light yet.

There is a question. Photons after the specular reflection are the result of the occurrence of atomic absorption of photons resulting transition to the high energy level and then back to low-energy-level emitting photons, or are the result of the occurrence of direct reflection of pinball? Because of the presence of the photoelectric effect, it is clearly that the later is not established. Therefore, the reflection process of photon goes through the process that atomic absorption transitions to the high energy level and then transitions to the low-energy-level emitting photons. This process takes time. This shows that the method of reflection is not measuring the speed of light itself. It is the apparent velocity including the reflection process. The true speed of light is faster than the return speed of light measured by the reflection.

It can be seen from the measurement methods of the speed of light, even if the reflection time of light can be ignored, these methods can only measure speed relative to the light source or the reflector.

Photons will interact with matter when encounter substances. Whose action is weak will change the travel direction of photons. Whose action is strong will absorb the photons. It all depends on that when to emit, by which form to emit, or whether it also emit the same photons or not. The process that photons propagate within the material, is the process that photons interact with the matter, and is the process of absorb and re-emit of light. Because the speed and degree of different materials to the absorption of light and re-emitting are different, so transmission speed and degree of refraction, transmission and reflection are different when light travels in different media. In space, the speed of light relative to the light source has a relatively established range. As the 
light was absorbed and re-emitted by the medium (without space) in the process of transmission within the medium (without space), so propagation velocity of light relative to the medium (without space) in the medium (without space) is related to the nature of the medium, has little to do with the speed of the original light source, and also has a more defined scope. On the earth, the density of matter in vacuum (there are materials in vacuum. Vacuum is not equal to space.) is the lowest, the speed of light spreading in a vacuum is the fastest.

The current status of the speed of light measurement technology are: (1) There is no means of measuring the one-way speed of light; (2) The return-way speed of light measured by reflection method is not the true speed of light; (3) As the speed of light is too fast, there is no way to accurately measure the speed of light relative to the measuring instruments when there is relative motion of the light source relative to the equipment of measurement; (4) As the starlight was interfered with the universe material or absorbed and then be re-emitted during the journey to the earth. The speed of the starlight reaching the earth relative to the earth is similar to the speed of light emitted by a light source on the earth relative to the earth. Even if the invention of the one-way speed of light measuring instrument in the future, it is still difficult to observe the difference between the speed of starlight and the speed of light emitted by fixing lamp on the earth.

\section{The Nature of Field}

Field is formed of space and tiny particles distributed in space. Generally these tiny particles are still smaller than photons, and can be observed difficultly or can not be observed by light. So are electric field, magnetic field, and gravitational field.

\section{Light Source and Battery}

In a circuit formed of battery, lamp and wire, lamp emits light, emitting both mass and energy; batteries provides both energy and mass; the battery's own energy is reduced, the mass is also declining.

\section{Conclusions}

(1) The nature of light is particle, group photons behavior with characteristics similar to the wave.

(2) The speed of photons relative to light source is escape velocity of the photon.

(3) Currently there is no accurate method of measuring the speed of light.

(4) For the speed of starlight relative to the earth, the closer to the earth, the closer to the speed of light emitted by the light source on the earth relative to the earth.

(5) The field is composed of space and tiny particles.

\section{Acknowledgment}

The paper is supported by the National Natural Science Foundation of China (NSFC) (Grant No. 51374183 and 51490653).

\section{References}

10000 Science crux physics editorial committee. (2009). 10000 Science crux-physics volume. Beijing: Science Press. (in Chinese)

Fan, L. G., \& Feng, J. S. (2007). The nature of light. China Engineering Science, 9(2), 58-67. (in Chinese)

Kragh, H. S. (2006). Cosmologies with varying speed of light: A historical perspective. Studies in History and Philosophy of Modern Physics, 37, 726-737. http://dx.doi.org/10.1016/j.shpsb.2006.04.004

Li, Z. F. (2008). Special relativity arising from a misunderstanding of experimental results on the constant speed of light. Physics Essays, 21(2), 96-102.

Li, Z. F., Li, T. J., Wang, C. J., Wang, Z. Y., \& Tian, X. M. (2007), The essence of special relativity and its influence on science, philosophy \& society. Scientific Inquiry, 8(2), 229-236.

Panarella, E. (1987). Nonlinear behavior of light at very low intensities: the "photon clump" model //William M. Honig, David M. Craft, Emilio Panarella. Quantum uncertainties recent and future experiments and interpretations. NATO ASI Series, Series B: Physics, 162, 105-167.

Smeulders, P. (2008). The measurement of the speed of light. Superlattices and Microstructures, 43, 651-654. http://dx.doi.org/10.1016/j.spmi.2007.07.007

\section{Copyrights}

Copyright for this article is retained by the author(s), with first publication rights granted to the journal.

This is an open-access article distributed under the terms and conditions of the Creative Commons Attribution license (http://creativecommons.org/licenses/by/3.0/). 